Pacific Journal of Mathematics

DEFORMING VARIETIES OF $\boldsymbol{k}$-PLANES OF PROJECTIVE 


\title{
DEFORMING VARIETIES OF $k$-PLANES OF PROJECTIVE COMPLETE INTERSECTIONS
}

\section{Ciprian Borcea}

\begin{abstract}
We consider the variety $F$ of $k$-dimensional linear projective subspaces lying on a generic projective complete intersection $S$. Under general assumptions involving $k$, the multidegree and the dimension of $S$, we prove that $F$ is connected, smooth, and its local deformations come from deformations of $S$.
\end{abstract}

Introduction. Linear varieties lying on a projective variety have been considered in several contexts.

A classical instance, going back to Cayley [6], is that of a smooth cubic surface. There are twenty-seven lines on such a surface, and, as observed later, the incidence preserving permutations of this set of lines form a group isomorphic to the Weyl group of a root system of type $E_{6}$. It is also the monodromy group of the global family of smooth cubics and the Galois group of the corresponding enumerative problem (see [12]).

Similar results (involving the root system $D_{2 k+3}$ ) hold for the $k$ planes contained in a smooth $2 k$-dimensional intersection of two quadrics $([14,16])$.

Beyond the enumerative level, and besides homogeneous-rational varieties such as Grassmannians or linear spaces lying on a smooth quadric, a first example should be the Fano surface of lines contained in a cubic threefold ([11]). The Abel-Jacobi map induces an isomorphism from the Albanese variety of the Fano surface to the intermediate Jacobian of the cubic threefold and one has a global Torelli theorem $([7,19])$.

With planes instead of lines, but generically this time, the analogous statements hold true for cubic fivefolds $([8,10])$.

Nor should cubic fourfolds be neglected here: their varieties of lines are irreducible symplectic projective fourfolds ([3]) which play an important role in the proof of the global Torelli theorem ([20]).

We also mention the variety of $k$-planes contained in a smooth $(2 k+1)$-dimensional intersection of two quadrics: it is an Abelian variety isomorphic with the intermediate Jacobian of the given intersection of quadrics $([9,16])$. 
All these varieties may be realized as zero loci of sections of certain homogeneous vector bundles over Grassmannians $([\mathbf{1}, \mathbf{1 8}])$. This circumstance makes the Schubert calculus relevant, for instance, in computing Chern numbers; it also reduces questions about connectivity, regularity, etc., as well as deformations to questions about the cohomology of homogeneous vector bundles.

Our main concern will be to set up a general framework for a calculus with weights, such that the theorem of Bott [5] become expressive in this context-a perspective we initially used in [4].

Specific computations enabled Wehler to deal with small deformations of Fano surfaces: he showed, namely, that all of them are induced by deformations of the corresponding cubic threefolds ([21]). This result is here extended to a large class (Theorem 5.3). Similarly (Theorem 4.1), we extend (and give an alternative proof for) the connectedness result of Barth and Van de Ven concerning lines on hypersurfaces ([2]).

1. Varieties of $k$-planes. We shall consider projective $k$-planes contained in a complete intersection $S=S_{n}(d)$ of dimension $n$ and multidegree $d=\left(d_{1}, \ldots, d_{r}\right)$ in the projective space $P=P_{n+r}$ over the complex field $C$.

Let $\mathscr{O}_{P}(m)$ denote the $m$ th tensor power of the hyperplane line bundle on $P$ and let $S$ be given as the variety of zeros $Z(s)=S$ of a section $s \in H^{0}(P, E)$, where $E=\bigoplus_{t=1}^{r} \mathscr{O}_{P}\left(d_{t}\right)$.

Denote by $G=G(k+1, n+r+1)$ the Grassmann variety of projective $k$-planes in $P$, i.e. $(k+1)$-planes in $C^{n+r+1}$, and let $\Gamma \subset P \times G$ be the subvariety defined by the incidence relation $\Gamma=\{(x, \pi) \mid x \in \pi\}$, with canonical projections:

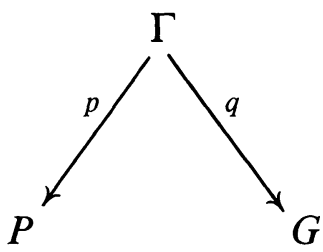

$p$ represents $\Gamma$ as a $G(k, n+r)$-bundle over $P$ and $q$ represents $\Gamma$ as a $P_{k}$-bundle over $G$. Accordingly, we have isomorphisms: $H^{0}(P, E)$ $\stackrel{\sim}{\rightarrow} H^{0}\left(\Gamma, p^{*} E\right) \stackrel{\sim}{\rightarrow} H^{0}\left(G, q_{*} p^{*} E\right)$.

If $0 \rightarrow \tau=\tau_{k+1} \rightarrow G \times C^{n+r+1} \rightarrow Q=Q_{n+r-k} \rightarrow 0$ denotes the canonical exact sequence of vector bundles over the Grassmannian $G$, we have a natural identification: $q_{*} p^{*} \mathscr{O}_{P}(m)=S^{m}\left(\tau^{*}\right)=$ the $m$ th symmetric tensor power of the dual tautological bundle. 
Put $\mathscr{E}=q_{*} p^{*} E$.

Let $\Phi$ be the isomorphism indicated above:

$$
\Phi: H^{0}(P, E) \stackrel{\sim}{\rightarrow} H^{0}(G, \mathscr{E})=\bigoplus_{t=1}^{r} H^{0}\left(G, S^{d_{t}}\left(\tau^{*}\right)\right) .
$$

To $s \in H^{0}(P, E)$, defining the variety $Z(s)=S$, we thus associate $\Phi(s) \in H^{0}(G, \mathscr{E})$, defining the variety of zeros $Z(\Phi(s))=F_{k}(S)=F$, which consists of all $k$-planes contained in $S \subset P$.

REMARK 1.1. The rank of $\mathscr{E}$ is $\sum_{t=1}^{r}\left(\begin{array}{c}d_{t}+k \\ k\end{array}\right)$, and we expect $F$ to be non-empty for $\operatorname{dim} G-\operatorname{rk} \mathscr{E} \geq 0$, i.e. for

$$
(k+1)(n+r-k)-\sum_{t=1}^{r}\left(\begin{array}{c}
d_{t}+k \\
k
\end{array}\right) \geq 0 .
$$

This will presently be seen to be true, provided $S$ is not a quadric, in which case the assumption $n \geq 2 k$ is needed. Note that, if $S$ is neither a quadric, nor a linear space, condition $\left(\mathrm{A}_{0}\right)$ already implies $n>2 k$.

2. Dimension and smoothness in the generic case. Let $V=H^{0}(P, E)$ and consider the subvariety $I \subset G \times V$ defined by: $I=\left\{(s, \pi)|s|_{\pi}=\right.$ $0\}$, with projections:

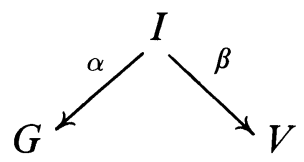

$\alpha$ represents $I$ as a sub-vector-bundle of $G \times V \rightarrow G$, which shows that $I$ is smooth, while $\beta$ is proper and the fibre over $s \in V$ is precisely $Z(\Phi(s))$.

Confirming our Remark 1.1, we have:

Proposition 2.1. If $\operatorname{dim} G-\mathrm{rk} \mathscr{E} \geq 0, \beta$ is onto, provided $n \geq 2 k$ in the case of quadrics.

Proof. If we find a $k$-plane $\pi$ in $S$, with $S$ smooth along $\pi$, and such that the normal bundle $N_{\pi / S}$ has $H^{1}\left(\pi, N_{\pi / S}\right)=0$, the proposition will follow from Kodaira's criterion for stability of compact submanifolds [15].

We consider the exact sequence:

$$
\left.0 \rightarrow N_{\pi / S} \rightarrow N_{\pi / P} \rightarrow N_{S / P}\right|_{\pi} \rightarrow 0 .
$$


We have:

$$
N_{\pi / P}=\bigoplus^{n+r-k} \mathscr{O}_{\pi}(1) \text { and }\left.N_{S / P}\right|_{\pi}=\bigoplus_{t=1}^{r} \mathscr{O}_{\pi}\left(d_{t}\right) .
$$

Let $\pi$ be given by $x_{k+1}=\cdots=x_{n+r}=0$, for homogeneous coordinates $\left(x_{0}: \cdots: x_{n+r}\right)$, so that $s \in H^{0}(P, E),\left.s\right|_{\pi}=0$ will be given by $r$ homogeneous polynomials $\left(s_{1}, \ldots, s_{r}\right)$ of the form

$$
s_{t}=\sum_{i=k+1}^{n+r} x_{i} \cdot p_{t}^{(i)}+r_{t}
$$

where

$$
\begin{gathered}
p_{t}^{(i)}=\sum_{\mu} c_{t \mu}^{(i)} \cdot x^{\mu}, \\
\mu=\left(\mu_{0}, \ldots, \mu_{k}\right), x^{\mu}=x_{0}^{\mu} \cdots x_{k}^{\mu_{k}},|\mu|=\mu_{0}+\cdots+\mu_{k}=d_{t}-1
\end{gathered}
$$

and every monomial in $r_{t}$ contains a product $x_{i} x_{j}$ with $i \geq j>k$.

Since we may suppose $n \geq 2 k$, the condition that $S$ be smooth along $\pi$ is satisfied for generic $s$. (For example, the following matrix of partial derivatives

$$
\left(\frac{\partial s_{t}}{\partial x_{i}}(x)\right)_{i \geq k+1}, \quad x \in \pi
$$

may be produced:

$$
\left.\left(\begin{array}{cccccc}
x_{0}^{d_{1}-1} & \ldots & x_{k}^{d_{1}-1} & 0 & 0 & \ldots \\
0 & x_{0}^{d_{2}-1} & \ldots & x_{k}^{d_{2}-1} & 0 & \ldots \\
\ldots & \ldots & \ddot{d}_{r}-1 & \ldots & \ldots & \ldots \\
0 & 0 \ldots 0 & x_{0}^{d_{r}-1} & \ldots & \ldots & x_{k}^{r_{r}} \ldots
\end{array}\right)\right)
$$

We represent a global section of $N_{\pi / P}$ by a matrix

$$
a=\left(a_{i j}\right)_{0 \leq j \leq k<i \leq n+r},
$$

so that the map $H^{0}\left(N_{\pi / P}\right) \stackrel{\sigma}{\rightarrow} H^{0}\left(N_{S / P} \mid \pi\right)$ induced from (1) is described by

$$
A \rightarrow\left(\sum_{j \leq k<i} a_{i j} \cdot p_{t}^{(i)} \cdot x_{j}\right)_{1 \leq t \leq r} \in H^{0}\left(\bigoplus_{t=1}^{r} \mathscr{O}_{\pi}\left(d_{t}\right)\right) .
$$


Looking at monomial coefficients in (4) and using (3), one obtains that $\sigma$ is a surjection if and only if the linear system (with indeterminates $a_{i j}$ )

$$
\sum_{j \leq k<i} a_{i j} \cdot c_{t, \nu(j)}^{(i)}=0
$$

$$
t=1, \ldots, r, \nu=\left(\nu_{0}, \ldots, \nu_{k}\right),|\nu|=d_{t}
$$

where

$\nu(j)=\nu-(0, \ldots, 1,0, \ldots, 0)$ and $c_{t, \nu(j)}^{(i)}=0$ for $\nu(j)$ improper

has maximal rank, namely $\sum_{t=1}^{r}\left(\begin{array}{c}d_{t}+k \\ k\end{array}\right)=\mathrm{rk} \mathscr{E}=R$.

For generic $s$, this is actually the case. To see it, consider the lexicographic order on the set of column-indices $\{(i, j) \mid 0 \leq j \leq k<i \leq$ $n+r\}$ and look at the $R \times R$ matrix given by the first $R$ columns. Its determinant is a polynomial in $c_{t, \mu}^{(i)}$, with $|\mu|=d_{t}-1$. It is not difficult to check that this polynomial is different from zero. Consider, for example, the lexicographic order on the set of indices $(i, t, \mu)$ affecting the coefficients $c_{t, \mu}^{(i)}$. Now order the monomials in the expression of the above determinant according to the rule: $m_{1}>m_{2}$ if the smallest index $(i, t, \mu)$ for which $c_{t, \mu}^{(i)}$ occurs in $m_{1}$ with exponent $p_{1}$ and in $m_{2}$ with exponent $p_{2} \neq p_{1}$, we have $p_{1}>p_{2}$. The greatest monomial in this ordering will have perforce coefficient 1 or -1 , since in each row, the choice of $c_{t, \mu}^{(i)}$ entering this monomial is prescribed.

Thus, for generic $s, S$ is smooth along $\pi$ and $H^{1}\left(\pi, N_{\pi / S}\right)=0$.

COROLlARY 2.2. The projective $k$-planes contained in a generic complete intersection $S_{n}(d)$ of dimension $n$ and multidegree $d=$ $\left(d_{1}, \ldots, d_{r}\right)$ in $P_{n+r}$ define a smooth subvariety $F_{k}\left(S_{n}(d)\right)$ of $G(k+1, n+r+1)$ of codimension $\sum_{t=1}^{r}\left(\begin{array}{c}d_{t}+k \\ k\end{array}\right)$, provided that $(k+1)(n+r-k) \geq \sum_{t=1}^{r}\left(\begin{array}{c}d_{t}+k \\ k\end{array}\right)$ and $S_{n}(d)$ is not quadric, in which last case $n \geq 2 k$ is required.

REMARK 2.3. The variety of lines $F_{1}\left(S_{n}(3)\right)$ of a cubic hypersurface $S_{n}(3) \subset P_{n+1}$ is smooth if the cubic is smooth, but in general, the smoothness of $S_{n}(d)$ does not imply that of $F_{k}\left(S_{n}(d)\right)$ (cf. [12], [18]).

3. Weights. In what follows, we take $\operatorname{dim} G \geq \operatorname{rk} \mathscr{E}$ (and $n \geq 2 k$ for quadrics), and assume the complete intersection $S=S_{n}(d)$ to be 
such that the codimension of $F=F_{k}(S)$ in $G=G(k+1, n+r+1)$ be precisely rk $\mathscr{E}$. Generically, this is the case (Corollary 2.2).

Let $J_{F}$ denote the sheaf of ideals defining $F$ on $G$.

The Koszul complex of (the section of $\mathscr{E}=q_{*} p^{*} E$ defining) $J_{F}$ gives, for any holomorphic vector bundle $M$ on $G$, spectral sequences:

$$
\begin{aligned}
& H^{p}\left(G, M \otimes \bigwedge^{q} \mathscr{E}^{*}\right) \Rightarrow H^{p-q}\left(F,\left.M\right|_{F}\right), \\
& H^{p}\left(G, M \otimes \bigwedge^{q+1} \mathscr{E}^{*}\right) \Rightarrow H^{p-q}\left(G, M \otimes J_{F}\right), \quad q \geq 0 .
\end{aligned}
$$

If $M$ is a homogeneous vector bundle, we may use the theorem of Bott [5, Th. IV'] for dealing with the groups on the left. To this purpose, we use the following description of the Grassmann manifold $G(k+1, n+r+1)$ :

$\mathrm{SL}(n+r+1, C)$, which is the universal cover of $\operatorname{Aut}\left(P_{n+r}\right)=$ $\operatorname{PGL}(n+r+1, C)$, has Lie algebra $\operatorname{sl}(n+r+1, C)=\left\{A=\left(a_{i j}\right) \mid \operatorname{tr} A=\right.$ $0\}$. Take as Cartan subalgebra $h=\left\{A \mid a_{i j}=0\right.$ for $\left.i \neq j\right\}$. This gives root spaces $L_{i j}=C \cdot E_{i j}(i \neq j)$ where $E_{i j}$ has zeros everywhere except the $(i, j)$ entry.

The Killing form identifies the corresponding roots $\alpha_{i j}$ with $E_{i i}-$ $E_{j j} \quad(i \neq j)$ so that the root system $A_{n+r}$ may be viewed as embedded in a euclidean space with orthonormal basis $e_{i}=E_{i i}, i=1, \ldots$, $n+r+1$, the roots being represented by vectors $\alpha$ orthogonal to $e_{1}+\cdots+e_{n+r+1}$ and of square-norm $(\alpha, \alpha)=2$ (cf. [36, p. 64]).

Put $\alpha_{s}=\alpha_{s+1, s}=e_{s+1}-e_{s} .\left\{\alpha_{s} \mid s=1, \ldots, n+r\right\}$ gives a basis of the root system $A_{n+r}$.

If $U_{k+1}$ denotes the subgroup of $\mathrm{SL}(n+r+1, C)$ consisting of the transformations which preserve the linear space $\left\{x_{k+2}=\cdots=\right.$ $\left.x_{n+r+1}=0\right\} \subset C^{n+r+1}$ with coordinates $\left(x_{1}, \ldots, x_{n+r+1}\right)$, the Lie algebra $u_{k+1}$ of $U_{k+1}$ will contain $h$, all the negative roots $\left(\alpha_{i j}, i<\right.$ $j$ ) and all positive roots not involving $\alpha_{k+1}$ when expressed in terms of the given basis.

We have $G(k+1, n+r+1)=\operatorname{SL}(n+r+1, C) / U_{k+1}$, which is the description we shall use.

Let us now investigate the weights associated to various homogeneous vector bundles over $G=G(k+1, n+r+1)$.

Such a bundle is defined by a holomorphic representation $\rho: U_{k+1}$ $\rightarrow \mathrm{GL}(N, C)$ and the weights are taken with respect to $h$.

(a) Consider first the tautological bundle $\tau$ over $G$. It corresponds 
to the natural representation of $U_{k+1}$ on the invariant subspace $\left\{x_{k+2}\right.$ $\left.=\cdots=x_{n+r+1}=0\right\}$.

Let $\beta_{s}$ denote the weight characterized by

$$
\left(\beta_{s}, \alpha_{t}\right)=0 \text { for } t \neq s \text { and }\left(\beta_{s}, \alpha_{s}\right)=\frac{1}{2}\left(\alpha_{s}, \alpha_{s}\right)=1 .
$$

An elementary computation then gives the weights of

$$
\tau_{k+1}: t_{1}=-\beta_{1}, t_{2}=\beta_{1}-\beta_{2}, \ldots, t_{k+1}=\beta_{k}-\beta_{k+1} \text {. }
$$

(b) The line bundle $\operatorname{det}\left(\tau_{k+1}^{*}\right)$, which gives the Plücker embedding of $G(k+1, n+r+1)$, has therefore associated weight: $\beta_{k+1}$.

(c) The tangent bundle of $G: \theta_{G}$ is given by the adjoint representation of $U_{k+1}$ on $\operatorname{sl}(n+r+1, C) / u_{k+1}$. Consequently, its weights are precisely the positive roots involving $\alpha_{k+1}$ in their expression, namely $\alpha_{i j}, i>k+1 \geq j$.

(d) $\mathscr{E}^{*}=\bigoplus_{m=1}^{r} S^{d_{m}}\left(\tau_{k+1}^{*}\right)^{*}$ and (a) immediately gives that its weights are of the form:

$$
\sum_{i=1}^{k+1} a_{i} t_{i}=\left(a_{2}-a_{1}\right) \beta_{1}+\left(a_{3}-a_{2}\right) \beta_{2}+\cdots+\left(a_{k+1}-a_{k}\right) \beta_{k}-a_{k+1} \beta_{k+1}
$$

with $a_{i} \in N, \sum_{i=1}^{k+1} a_{i}=d_{m}$ for some $m \leq r$.

We now draw up a table of scalar products of positive roots and various weights, which will be relevant in estimating indices of weights.

$\delta$ is half the sum of all positive roots.

$\omega=\sum_{i=1}^{k+1} a_{i} t_{i}, a_{i} \in Z$ (motivated by (d) above and the spectral sequences (6)).

$$
1 \leq m \leq k \text {. }
$$

We anticipate here the type of reasoning to be used in the sequel. Given a homogeneous vector bundle over $G$, defined by a representation $U_{k+1} \rightarrow \mathrm{GL}(N, C)$, we first produce a filtration with consecutive quotients corresponding to irreducible representations of $U_{k+1}$. Such an irreducible representation determines a highest weight, say $\rho$. This $\rho$ has to be one of the weights of the original representation and further satisfy $\left(\rho, \alpha_{s}\right) \geq 0$ for all $s \neq k+1$.

In our computations $\rho$ will be either of type $\omega$ or $\omega+\alpha_{n+r+1, m}$ $(m \leq k+1)$.

In order to obtain the vanishing of $H^{s}(G, \rho)$, it will suffice either to ascertain the singularity of the weight $\rho+\delta$ or to prove: $s<$ index $(\rho+\delta)$.

In this context, the main feature of our table of products is that $\left(\alpha_{t, m}, \rho+\delta\right)$ increases by 1 when $t$ increases by 1 , except the last step for $\rho=\omega+\alpha_{n+r+1, m}(m \leq k+1)$. 
TABLe 1

\begin{tabular}{|c|c|c|c|c|c|}
\hline & Conditions & $\delta$ & $\omega$ & $\alpha_{n+r+1, m}$ & $\alpha_{n+r+1, k+1}$ \\
\hline$\alpha_{p}$ & $\begin{array}{l}p \neq m-1, m \\
p \leq k\end{array}$ & 1 & $a_{p+1}-a_{p}$ & 0 & $\begin{array}{l}p<k \quad 0 \\
p=k\end{array}$ \\
\hline $\begin{array}{l}\alpha_{m-1} \\
\alpha_{m}\end{array}$ & & $\begin{array}{l}1 \\
1\end{array}$ & $\begin{array}{l}a_{m}-a_{m-1} \\
a_{m+1}-a_{m}\end{array}$ & $\begin{array}{r}-1 \\
1\end{array}$ & $\begin{array}{r}0 \\
m<k \quad 0 \\
m=k-1\end{array}$ \\
\hline$\alpha_{q}$ & $k+1 \leq q \leq n+r$ & 1 & 0 & $\begin{array}{ll}q<n+r & 0 \\
q=n+r & 1\end{array}$ & $\begin{array}{l}0 \\
1 \\
\end{array}$ \\
\hline$\alpha_{t, k+1}$ & $t>k+1$ & $t-k-1$ & $-a_{k+1}$ & $\begin{array}{ll}t<n+r+1 & 0 \\
t=n+r+1 & 1\end{array}$ & $\begin{array}{l}1 \\
2 \\
\end{array}$ \\
\hline$\alpha_{t, m}$ & $t>k+1$ & $t-m$ & $-a_{m}$ & $\begin{array}{ll}t<n+r+1 & 1 \\
t=n+r+1 & 2\end{array}$ & $\begin{array}{l}0 \\
1\end{array}$ \\
\hline$\alpha_{t, p}$ & $\begin{array}{l}t>k+1>p \\
p \neq m\end{array}$ & $t-p$ & $-a_{p}$ & $\begin{array}{ll}t<n+r+1 & 0 \\
t=n+r+1 & 1\end{array}$ & $\begin{array}{l}0 \\
1\end{array}$ \\
\hline
\end{tabular}

Note also that for $1 \leq p \leq k+1,\left(\alpha_{k+2, p}, \rho+\delta\right)<\left(\alpha_{k+2, p-1}, \rho+\delta\right)$ since $\left(\alpha_{p-1}, \rho\right) \geq 0$.

\section{Connectedness. Suppose}

$$
\operatorname{dim} F=\operatorname{dim} G-\text { rk } \mathscr{E} \geq 1
$$

$F$ is connected if and only if $H^{0}\left(\mathscr{O}_{F}\right)=C$.

We have $H^{s}\left(G, \bigwedge^{s} \mathscr{E}^{*}\right) \Rightarrow H^{0}\left(\mathscr{O}_{F}\right)$; therefore the vanishing of $H^{s}\left(G, \bigwedge^{s} \mathscr{E}^{*}\right)$ for $s>0$ will imply the connectedness of $F$.

According to our method, described at the end of $\S 3$, we examine $H^{s}(G, \rho)$, with $\rho$ an irreducible representation of $U_{k+1}$ with highest weight (again denoted $\rho$ ) among the weights of $\Lambda^{s} \mathscr{E}^{*}$. Thus $\rho=$ $\omega=\sum_{i=1}^{k+1} a_{i} t_{i}$ and we know (see Table 1):

(1) $a_{k+1} \geq a_{k} \geq \cdots \geq a_{q} \geq 0$;

(2) $\rho+\delta$ is either singular or of index $u(n+r-k), 1 \leq u \leq k$ $(u=k+1$ is excluded because rk $\mathscr{E}<\operatorname{dim} G)$.

Suppose therefore $s=u(n+r-k)$.

For $\rho+\delta$ to have index $s$, we must have $\left(\alpha_{t, p}, \rho+\delta\right)>0$ for $p=1, \ldots, k+1-u$; in particular: $a_{k+1-u} \leq u$.

Now remember that $\rho$ is a weight of $\Lambda^{s} \mathscr{E}^{*}$, thus a sum of $s$ weights of $\mathscr{E}^{*}$, each weight counted at most as many times as the 
dimension of its eigenspace. There are (multiplicities included) $\sum_{m=1}^{r}\left(\begin{array}{c}d_{m}+u-1 \\ u-1\end{array}\right)$ weights involving only $t_{i}, i>k+1-u$. Adding any other weight increases some $a_{j}, j \leq k+1-u$; thus we must not add more than $u(k+1-u)$ such weights. This will be clearly impossible if $n$ satisfies the following conditions:

$$
\sum_{m=1}^{r}\left(\begin{array}{c}
d_{m}+u-1 \\
u-1
\end{array}\right)+u(k+1-u)<u(n+r-k)=s
$$

with $u$ running from 1 to $k$.

Now, use (repeatedly) the formula:

$$
\frac{1}{q+1}\left(\begin{array}{c}
d_{m}+q \\
q
\end{array}\right)-\frac{1}{q}\left(\begin{array}{c}
d_{m}+q-1 \\
q-1
\end{array}\right)=\frac{d_{m}-1}{q(q+1)}\left(\begin{array}{c}
d_{m}+q-1 \\
q-1
\end{array}\right)
$$

to show that if some $d_{m} \geq 3$, or at least two degrees in $d$ are $\geq 2$, then $\left(\mathrm{C}_{u}\right), 1 \leq u \leq k$, is a consequence of our assumption $\left(\mathrm{A}_{1}\right)$. Note that $\left(\mathrm{C}_{1}\right)$ reads: $n>2 k$.

We have therefore:

THEOREM 4.1. Let $S=S_{n}\left(d_{1}, \ldots, d_{r}\right)$ be a complete intersection in $P_{n+r}$ and $F=F_{k}(S)$ its variety of projective $k$-planes. Suppose

$$
\operatorname{dim} F=(k+1)(n+r-k)-\sum_{m=1}^{r}\left(\begin{array}{c}
d_{m}+k \\
k
\end{array}\right) \geq 1,
$$

or, in case $S$ is a quadric, suppose $n>2 k$.

Then $F$ is connected.

REMARK 4.2. For a smooth quadric $S=S_{2 k}(2), F_{k}(S)$ consists of two isomorphic (hermitian symmetric) connected components.

This should rather be viewed as the exception which confirms the rule: $S_{2 k}(2)$ is a homogeneous (hermitian symmetric) space (of rank one) in its own right, and the generating $k$-planes of the two families in $F_{k}(S)$ correspond to Schubert cycles which are not homologically equivalent.

REMARK 4.3. There is a simple formula for the canonical bundle of $F=F_{k}\left(S_{n}(d)\right)$, when smooth.

Let $\mathscr{O}_{G}(1)$ denote the positive generator of $\operatorname{Pic}(G)$, restricting to $\mathscr{O}_{F}(1)$ on $F$.

Set

Then $K_{F}=\mathscr{O}_{F}(K)$.

$$
K=\sum_{m=1}^{r}\left(\begin{array}{c}
d_{m}+k \\
k+1
\end{array}\right)-(n+r+1) .
$$


5. Deformations. In this section we assume that $F=F_{k}\left(S_{n}(d)\right)$ has the "right" codimension and dimension at least two:

$$
\operatorname{dim} F=\operatorname{dim} G-\text { rk } \mathscr{E} \geq 2 .
$$

Our purpose is to produce conditions on $(n, d, k)$ which ensure the completeness of the natural deformation of $F$, parametrized by a neighborhood of the section $\Phi(s) \in H^{0}(G, \mathscr{E})$ defining $F$. Notice that the family of complete intersections to which $S_{n}(d)$ belongs (parametrized by a neighbourhood of $s \in H^{0}(P, E) \cong H^{0}(G, \mathscr{E})$, i.e. the "same" base) is itself complete (see [4], [17], [21]).

A sufficient condition for completeness is the vanishing of $H^{1}\left(G, \mathscr{E} \otimes J_{F}\right)$ and $H^{1}\left(F,\left.\theta_{G}\right|_{F}\right)$. This is a general result for varieties defined by sections in a vector bundle (see [21]).

We look therefore at the spectral sequences (6) abutting to the above two groups.

(5.1) Take first $H^{s}\left(G, \mathscr{E} \otimes \bigwedge^{s} \mathscr{E}^{*}\right), s \geq 1$.

We obtain vanishing conditions for these groups as we did for $H^{s}\left(G, \bigwedge^{s} \mathscr{E}^{*}\right)$ in $\S 4$.

Let $D=\max _{1 \leq m \leq r}\left(d_{m}\right)$. Filtering and taking highest weights will produce as above weights $\rho=\omega=\sum_{i=1}^{k+1} a_{i} t_{i}$, with $\left(\alpha_{p}, \rho\right) \geq 0$ for $p \leq k$.

Since $\rho$ is the sum of a weight $\omega^{\prime}$ of $\mathscr{E}$ and a weight $\omega^{\prime \prime}$ of $\bigwedge^{s} \mathscr{E} *$, adding $\omega^{\prime}$ to $\omega^{\prime \prime}=\sum_{i=1}^{k+1} a_{i}^{\prime \prime} t_{i}$ decreases some of its coefficients $a_{i}^{\prime \prime}$, diminishing their sum by at most $D$.

This means that our sufficient conditions $\left(\mathrm{C}_{u}\right), 1 \leq u \leq k$, for the vanishing of $H^{s}\left(G, \bigwedge^{s} \mathscr{E}^{*}\right), s \geq 1$, become, by the same type of reasoning, sufficient conditions $\left(\mathrm{C}_{u}^{D}\right), 1 \leq u \leq k$, for the vanishing of $H^{s}\left(G, \mathscr{E} \otimes \wedge^{s} \mathscr{E}^{*}\right)$, once we add $D$ to the left hand side of each inequality:

$$
\left(C_{u}^{D}\right) \quad \sum_{m=1}^{r}\left(\begin{array}{c}
d_{m}+u-1 \\
u-1
\end{array}\right)+u(k+1-u)+D<u(n+r-k) .
$$

(5.2) Consider now $H^{s+1}\left(G, \theta_{G} \otimes \bigwedge^{s} \mathscr{E}^{*}\right), s \geq 0$. For $s=0$, we have $H^{1}\left(G, \theta_{G}\right)=0$, because $G$ is rigid [5]. Suppose $s \geq 1$.

Again, using a filtration (actually, the representations we are dealing with are all completely reducible) and successive quotients corresponding to irreducible representations of $U_{k+1}$, we find that the highest weight $\rho$ associated to such a representation is necessarily of the form $\rho=\omega+\alpha_{t, m}$, with $\omega=\sum_{i=1}^{k+1} a_{i} t_{i}$ a weight of $\Lambda^{s} \mathscr{E}^{*}$, 
$t>k+1 \geq m$ (cf. $\S 3$ (c)), and further conditions: $\left(\rho, \alpha_{q}\right) \geq 0$ for all $q \neq k+1$, which imply in particular $t=n+r+1$.

Take therefore $\rho=\omega+\alpha_{n+r+1, m}(m \leq k+1)$ and consider the series of integers: $\left(\rho+\delta, \alpha_{t, p}\right)$ with $p \leq k+1$ fixed and $t$ increasing from $k+2$ to $n+r+1$. If $\rho+\delta$ is non-singular, this series of nonzero integers will keep the same sign, except possibly at the last step $t=n+r+1$, when it might "jump" precisely over zero (see Table 1).

Now let $p$ decrease from $k+1$ to 1 and notice the relations of the starting values in each series:

$$
\left(\rho+\delta, \alpha_{k+2, k+1}\right)<\left(\rho+\delta, \alpha_{k+2, k}\right)<\cdots<\left(\rho+\delta, \alpha_{k+2,1}\right) .
$$

This means that we might encounter non-vanishing cohomology $H^{s+1}(G, \rho)$ at most for $s+1$ or $s$ a multiple of $n+r-k$, say $u(n+r-k) \quad(u<k+1$ by our assumption $\operatorname{rk} \mathscr{E} \leq \operatorname{dim} G-2)$.

For the coefficients $a_{i}$ in $\omega=\sum_{i=1}^{k+1} a_{i} t_{i}$, we have either:

(1) $a_{k+1}>a_{k} \geq \cdots \geq a_{1}$ for $m=k+1$, or

(2) $a_{k+1} \geq \cdots \geq a_{m+1} ; a_{m+1}+1 \geq a_{m}>a_{m-1} \geq \cdots \geq a_{1}$ for $m \leq k$.

Since $\omega$ is a weight of $\bigwedge^{s} \mathscr{E}^{*}$, it appears that $\left(\mathrm{C}_{u}^{2}\right)$ above is a sufficient condition for the vanishing of $H^{s+1}(G, \rho)$.

Now, one may verify that the combination of $\left(\mathrm{A}_{2}\right)$ and $\left(\mathrm{C}_{1}^{D}\right)$ above implies $\left(\mathrm{C}_{u}^{D}\right)$ for $1 \leq u \leq k$.

First, suppose $d_{m} \geq 2$, which is no restriction of generality. Making use of the identity (7) in $\S 4$ and the fact that the right hand side in (7) clearly increases with $q$, the following implications obtain:

(i) If $k \geq 2,\left(\mathrm{~A}_{2}\right) \Rightarrow\left(\mathrm{C}_{k}^{D}\right)$ as soon as $\sum_{m=1}^{r}\left(d_{m}^{2}-1\right)>3 D+2$, i.e. $d \neq(2),(2,2),(3),(2,3)$; and for $n>6$ also for $d=(2,3)$.

(ii) If $u>1,\left(\mathrm{C}_{u+1}^{D}\right) \Rightarrow\left(\mathrm{C}_{u}^{D}\right)$ for $\sum_{m=1}^{r}\left(d_{m}^{2}-1\right) \geq D+6$, i.e. $d \neq(2),(2,2),(3)$.

Finally, for $d=(2),(2,2),(3)$ or $(2,3)$, a direct check shows that $\left(\mathrm{A}_{2}\right) \&\left(\mathrm{C}_{1}^{D}\right) \Rightarrow\left(\mathrm{C}_{u}^{D}\right)$.

Summing-up, we obtain:

THEOREM 5.3. Let $S=S_{n}\left(d_{1}, \ldots, d_{r}\right)$ be a complete intersection in $P_{n+r}$ and suppose that its variety of $k$-planes $F=F_{k}(S)$ satisfies

$$
\operatorname{dim} F=(k+1)(n+r-k)-\sum_{m=1}^{r}\left(\begin{array}{c}
d_{m}+k \\
k
\end{array}\right) \geq 2 .
$$

If $n>2 k+D$, where $D=\max _{1 \leq m \leq r}\left(d_{m}\right)$, then every small deformation of $F$ is induced by a (small) deformation of $S$. 


\section{REFERENCES}

[1] A. B. Altman and S. Kleiman, Foundations of the theory of Fano schemes, Compositio Math., 34 (1977), 3-47.

[2] W. Barth and A. Van de Ven, Fano varieties of lines on hypersurfaces, Archiv der Math., 31 (1978), 96-104.

[3] A. Beauville and R. Donagi, The variety of lines of a cubic fourfold, C. R. Acad. Sci. Paris, Ser. 1, 301 (1985), 703-706.

[4] C. Borcea, Smooth global complete intersections in certain compact homogeneous complex manifolds, J. reine u. angewandte Math., 344 (1983), 65-70.

[5] R. Bott, Homogeneous vector bundles, Ann. of Math., 66 (1957), 203-248.

[6] A. Cayley, A memoir on cubic surfaces, Phil. Trans. Royal Soc. London, CLIX (1869), 231-326.

[7] H. Clemens and Ph. Griffiths, The intermediate Jacobian of the cubic threefold, Ann. of Math., 95 (1972), 281-356.

[8] A. Collino, The Abel-Jacobi isomorphism for cubic fivefolds, Pacific J. Math., 122 (1986), 43-56.

[9] R. Donagi, Group law on the intersection of two quadrics, Annali Sc. N. Sup. Pisa, 7 (1980), 217-239.

[10] _ Generic Torelli for projective hypersurfaces, Compositio Math., 50 (1983), 325-353.

[11] G. Fano, Sul sistema $\infty^{2}$ di rette contenuto in una varietà cubica generale dello spazio a quattro dimensioni, Atti Reale Accad. Sci. Torino, 39 (1904), 778-792.

[12] J. Harris, Galois groups of enumerative problems, Duke Math. J., 46 (1979), 685-724.

[13] J. E. Humphreys, Introduction to Lie Algebras and Representation Theory, Springer-Verlag, New York-Heidelberg-Berlin, 1972.

[14] H. Knörer, Isolierte Singularitäten von Durchschnitten zweier Quadriken, Bonner Math. Schriften, 117 (1980).

[15] K. Kodaira, On stability of compact submanifolds of complex manifolds, Amer. J. Math., 85 (1963), 79-94.

[16] M. Reid, Cambridge Thesis, June 1972.

[17] E. Sernesi, Small deformations of global complete intersections, Boll. Un. Mat. Ital. (4), 12 (1975), 138-146.

[18] B. R. Tennison, On the quartic threefold, Proc. London Math. Soc., 29 (1974), 714-734.

[19] A. N. Tjurin, The geometry of the Fano surface of a non-singular cubic $F \subset P_{4}$ and Torelli theorems for Fano surfaces and cubics, Izv. Akad. Nauk SSSR. Ser. Mat., 35 (1971), 458-529 = Math. USSR Izv., 5 (1971), 517-546.

[20] C. Voisin, Théorème de Torelli pour les cubiques de $P_{5}$, Invent. Math., 86 (1986), 577-601.

[21] J. Wehler, Deformation of varieties defined by sections of homogeneous vector bundles, Math. Ann., 268 (1984), 519-532.

Received July 12, 1988.

National Institute for Scientific and Technical Creation

BD. PĂCII 220, 79622 BuCharest, RoMANIA 


\section{PACIFIC JOURNAL OF MATHEMATICS EDITORS}

\author{
V. S. VARADARAJAN \\ (Managing Editor) \\ University of California \\ Los Angeles, CA 90024-1555-05 \\ Herbert Clemens \\ University of Utah \\ Salt Lake City, UT 84112 \\ Thomas ENRIGHT \\ University of California, San Diego \\ La Jolla, CA 92093
}

R. FINN

Stanford University

Stanford, CA 94305

Hermann FlaschKa

University of Arizona

Tucson, AZ 85721

VAughan F. R. Jones

University of California

Berkeley, CA 94720

Steven Kerckhoff

Stanford University

Stanford, CA 94305
RobION KIRBY

University of California

Berkeley, CA 94720

C. C. MOore

University of California

Berkeley, CA 94720

HAROLd STARK

University of California, San Diego La Jolla, CA 92093

\begin{tabular}{|c|c|c|c|c|}
\hline & ASSOCIA & E EDI & & \\
\hline R. ARENS & $\begin{array}{l}\text { E. F. BECKENBACH } \\
(1906-1982)\end{array}$ & NeumanN & $\begin{array}{c}\text { F. WoLF } \\
(1904-1989)\end{array}$ & K. Yoshida \\
\hline & SUPPORTINC & INSTI & TIONS & \\
\hline UNIVERSITY & ARIZONA & UNIVERS & OF OREGON & \\
\hline UNIVERSITY & BRITISH COLUMBIA & UNIVER & OF SOUTHEI & CALIFORNIA \\
\hline CALIFORNIA & STITUTE OF TECHNOLOGY & STANFOI & UNIVERSITY & \\
\hline UNIVERSITY & CALIFORNIA & UNIVER & OF HAWAII & \\
\hline MONTANA S & TE UNIVERSITY & UNIVER & OF TOKYO & \\
\hline UNIVERSITY & NEVADA, RENO & UNIVER & OF UTAH & \\
\hline NEW MEXIC & TATE UNIVERSITY & WA & N STATE UN & ERSITY \\
\hline OREGON ST & UNIVERSITY & & OF WASHINC & $\mathrm{ON}$ \\
\hline
\end{tabular}




\section{Pacific Journal of Mathematics}

\section{Vol. 143, No. $1 \quad$ March, 1990}

Walter Bergweiler, On the fix-points of composite functions $\ldots \ldots \ldots \ldots \ldots 1$ Aldo Biancofiore, Maria Lucia Fania and Antonio Lanteri, Polarized surfaces with hyperelliptic sections $\ldots \ldots \ldots \ldots \ldots \ldots \ldots \ldots \ldots$

Ciprian Borcea, Deforming varieties of $k$-planes of projective complete

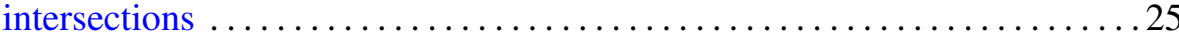

Morton Brown, Fixed points for orientation preserving homeomorphisms of

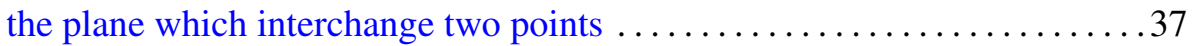

Hao Zhi Chuan, Note on the inequality of the arithmetic and geometric means

Paul Henry Edelman and Dennis E. White, Codes, transforms and the

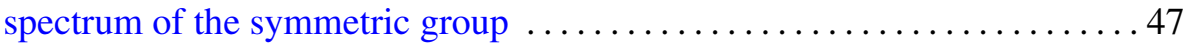

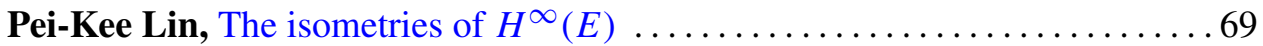

James J. Moloney, Residue class domains of the ring of convergent

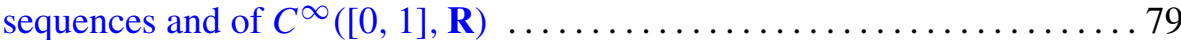

Zhong-Jin Ruan, The structure of pure completely bounded and completely

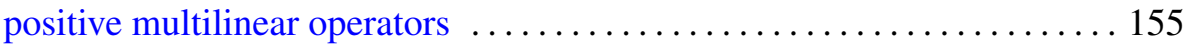

Wolfgang Ruess and William H. Summers, Weakly almost periodic semigroups of operators

Gideon Schwarz, A pretender to the title "canonical Moebius strip" . . . . . . 195

Ryszard Szwarc, Banach algebras associated with spherical representations of the free group 